\title{
EL CAMBIO LINGÜÍSTICO Y LA INTERFAZ SINTAXIS-PRAGMÁTICA: EVIDENCIAS DE LAS LENGUAS IBERO-ROMÁNICAS
}

\author{
Alice Corr \\ University of Cambridge \\ avc25@cam.ac.uk
}

This article analyses the function and distribution of the so-called "overt subject expletive" ello/ele/ell in certain non-standard Ibero-Romance varieties. Novel empirical facts are presented to illustrate the phenomenon's heterogeneous characterization, the present-day variation of which is argued to be explained by the different degrees of change undergone by the expletive element in each Ibero-Romance variety. The pragmatic import of today's Iberian expletive resembles the semantic value of the oldest and most commonly attested examples of the phenomenon in the historical corpora used. This observation serves as the point of departure for the diachronic reconstruction of the element's development from its proposed origin in epistemic contexts to the present day, where the alleged Iberian expletive exhibits both expletive and pragmatic characteristics even within the same variety.

Keywords: syntax-pragmatics interface, language change, overt expletive subjects, pragmaticization, Ibero-Romance languages

En este artículo se analiza la función y distribución del llamado "sujeto expletivo visible" ello/elelell en ciertas variedades ibero-románicas no estándares. Se 
presentan nuevos datos empíricos con el fin de demostrar la caracterización heterogénea del fenómeno, cuya variación actual, según se argumenta, se ve explicada por los distintos grados de cambio sufridos por el elemento expletivo en cada variedad ibero-románica. La contribución pragmática del expletivo ibérico actual semeja el valor semántico de las pruebas empíricas más antiguas y más frecuentes del elemento en los corpus históricos consultados, observación que sirve de punto de partida para reconstruir la trayectoria diacrónica del elemento desde su propuesto origen en contextos impersonales y epistémicos hasta la actualidad, en que el presunto expletivo ibérico demuestra características tanto expletivas como pragmáticas incluso dentro de la misma variedad.

Palabras clave: interfaz sintaxis-pragmática, cambio lingüístico, sujetos expletivos visibles, pragmatización, lenguas ibero-románicas

\section{INTRODUCCIÓN}

Es un truismo que el presente informa al pasado, y, al revés, que el pasado informa al presente; sin embargo, este tópico constituye la heurística que conduce el presente trabajo. Investigamos la presunta existencia de un sujeto expletivo visible en ciertas variedades ibero-románicas no estándar, defendiendo la hipótesis de que la caracterización y variación actual de dicho fenómeno se entiende mejor en términos de su desarrollo diacrónico. Este, a su vez, se ve explicado por las peculiaridades de la distribución y función del elemento como hoy se constata.

Asumimos que el cambio lingüístico puede interpretarse principalmente en términos de la adquisición del lenguaje, en el sentido de que los inputs lingüísticos vienen infradeterminados respecto a su valor paramétrico, convirtiéndose en una gramática interna basada en generalizaciones abducidas (Andersen 1973) por los niños al exponerse al entorno lingüís- 
tico. ${ }^{1}$ Estos no tienen acceso directo a las gramáticas internas subyacentes a las hablas de las que derivan su input lingüístico (Andersen 1973; Lightfoot 1979; Chomsky 1986), pues los niños forman a través de inferencias abductivas sus generalizaciones sobre los datos superficiales y visibles que les son directamente accesibles, de modo que la ambigüedad de los datos empíricos (y superficiales) sea condición necesaria para que se produzca el cambio lingüístico (Roberts 1993; 2007). Según los planteamientos generativistas (Chomsky 1981; 1986), los cambios incrementales en la parametrización que tengan lugar en el tiempo mediante el reanálisis del input lingüístico, pueden llevar a cambios "catastróficos" (Lightfoot 1997) dentro de una lengua a lo largo de los años. Desde esta óptica, el cambio gradual es mera apariencia, el resultado de varios reanálisis discretos que se acumulan con el tiempo, produciendo así el efecto del desarrollo diacrónico gradiente.

La presente investigación se centra en un subtipo del cambio lingüístico, a saber, la pragmatización, por medio de la cual un elemento lingüístico pasa por los mismos mecanismos genéricos que cualquier otro proceso de cambio diacrónico, diferenciándose en que dicho elemento adquiere valor pragmático en su papel funcional-interpretativo. En concreto, consideramos aquí que los procesos de la pragmatización y gramaticalización proceden de la misma manera (viz. ambos suponen el reanálisis de los patrones sintácticos que subyacen a los datos empíricos), aunque sus resultados reales sean diferentes y si bien al final los mecanis-

1 Para una crítica del uso del término "abducción” tal como se aplica en Andersen (1973) y trabajos posteriores, véanse Deutscher (2002) y Walkden (2011). 
mos exactos implicados en los dos procesos (p.e. la adquisición contra la pérdida de determinados rasgos formales) resulten distintos. En este artículo los consideraremos a la par, a fin de examinar sus consecuencias para el fenómeno del supuesto expletivo ibérico. No obstante, hay que resaltar las diferencias importantes que existen entre los dos.

Así pues, este trabajo tiene un doble objetivo: aportar nuevos datos sobre los llamados sujetos expletivos visibles en las lenguas ibero-románicas y ofrecer una narrativa alternativa de su desarrollo histórico, conforme a lo cual un elemento gramatical, a saber, el pronombre referencial ello, adquiere propiedades epistémicas mediante un proceso diacrónico de pragmatización. Concretamente, planteamos la hipótesis de que el uso no referencial de ello como "reserva-espacio" -es decir, su uso "expletivo"tiene su origen en la construcción epistémica y referencial ello es verdad cuando esta se halla en yuxtaposición con otra cláusula independiente seleccionada por el complementante que. Dicho brevemente, esta relación paratáctica se ve reanalizada como una subordinada y el pronombre ello va incorporando los matices subjetivos-epistémicos del contexto en que se encuentra, a través de un proceso de inferencia pragmática. Al asumir una semántica epistémica con independencia del contexto original, el pronombre ello, ya sin valor referencial, puede difundirse en nuevos entornos mediante el mecanismo productivo de la analogía.

En las páginas que siguen, se presentará primero una sinopsis teórica del fenómeno bajo investigación $(\$ 2)$, luego se expondrán los datos empíricos actuales junto con su caracterización formal (\$3), y, finalmente, se abordará someramente la reseña histórica del presunto expletivo ibérico desde su origen epistémico, tal como se propone aquí (\$4), has- 
ta su extensión a otros contextos, así como la diversificación de sus funciones que se observan hoy en día (\$5).

\section{AnteCedentes teóricos}

El fenómeno de los supuestos sujetos expletivos en las lenguas ibero-románicas ha sido objeto de interés para los lingüistas generativistas, debido al reto que parece suponer para la siguiente implicación universal: según la teoría generativa (Chomsky 1957 y siguientes), existen predicciones tipológicas muy fuertes respecto al parámetro de sujeto nulo y la realización visible de los sujetos expletivos (Rizzi 1982; 1986). Concretamente, se entiende que la presencia de los sujetos nulos referenciales supone la posibilidad de los sujetos expletivos nulos. Así pues, observamos que los sujetos expletivos son obligatorios en las lenguas que no permiten los sujetos referenciales nulos (el inglés y el francés, por ejemplo). Al contrario, las lenguas de sujeto nulo como el español, el portugués europeo o el catalán no admiten la realización abierta del sujeto expletivo y de ahí el contraste de gramaticalidad entre los ejemplos del español (a) y del inglés (b) en las siguientes construcciones: ${ }^{2}$

(1) a. (*Ello) nieva todos los días en el invierno.

b. *(It) snows everyday in winter.

${ }^{2}$ Hemos omitido aquí discusión de otras posibilidades paramétricas respecto a los sujetos nulos, por ejemplo las lenguas "semi" pro-drop, como el alemán (Biberauer 2009) o las lenguas de sujeto nulo "parcial" como el finlandés (Holmberg \& Nikanne 2002; Holmberg, Nayudu \& Sheehan 2009). 
(2) a. (*Ello) es evidente que el gobierno nuestro es corrupto.

b. ${ }^{*}(\mathrm{It})$ is evident that this government of ours is corrupt.

(3) a. i*Ello) hay una mosca en mi plato!

b. *(There) is a fly on my plate!

En la gramática generativa, se entiende por sujeto expletivo los pronombres impersonales y no referenciales que carecen de valor semántico alguno, ${ }^{3}$ los cuales aparecen en la posición canónica del sujeto, supuestamente para compensar la falta de sujeto argumental dentro de la estructura de la oración. ${ }^{4}$

Por eso, la observación del elemento pronominal ello, ell, ele (en adelante, se utilizará la etiqueta ELLo para referir a este fenómeno de manera genérica) en posición y función aparentemente expletiva en algunos dialectos ibero-románicos no estándar, como se ilustra en los ejemplos (4-6), plantea una situación problemática para nuestra comprensión

3 Tampoco tienen estatus argumental, es decir, que el verbo no les asigna un papel temático (excepto el expletivo de los predicados meteorológicos, el cual se considera un elemento cuasi-argumental debido a su capacidad de ligar un PRO en un adjunto (los ejemplos a seguir provienen de Fernández Soriano y Táboas 1999: 1746):

(i) Siempre llueve antes de [PRO nevar].

(ii) A veces nieva sin [PRO hacer frío].

(iii) Siempre oscurece después de [PRO llover].

${ }^{4}$ De ahí la postulación del Principio de Proyección Extendida (PPE) de Chomsky (1982), requisito que exige un sujeto preverbal en el especificador de T (EspecST), el núcleo flexivo de la jerarquía oracional. 
actual del parámetro de sujeto nulo y su tipología (Biberauer et al. 2009; Camacho 2013a):

(4) EsPañol dominicano (NO ESTÁNDAR)

¡Ello hay un búho en el techo!

(5) Catalán balear (no estándar)

Ell sembla que en Joan está malalt, pobret!

'(ELLO) parece que Juan está mal, el pobrecito'

(6) Portugués Europeo (NO ESTÁndar)

Ele já está nevando nesta altura do ano, que estranho!

'(ELLO) ya está nevando a estas alturas del año, ¡que raro!'

Estudios anteriores (Silva-Villar 1998; Carrilho 2005; 2008; Hinzelin \& Kaiser 2006; 2007; Martínez-Sanz 2011; Camacho 2013b; Muñoz Pérez 2014, etc.) tienden a asumir una caracterización homogénea del fenómeno en todas las variedades ibero-románicas. ${ }^{5}$ Aunque en ocasiones se propone que ELlo se halla en el ST (Muñoz Pérez 2014), por lo general se asume que el elemento está situado en el SC: o en una sola proyección (Hinzelin 2009) o en múltiples proyecciones (Silva-Villar 1998; Carrilho 2005), o incluso en ninguna proyección concreta (Bartra-Kaufmann 2011). En cambio, este artículo propone que se trata de un fenómeno

5 Para un resumen breve pero informativo de las posiciones teóricas tomadas por los varios autores que han trabajo sobre el fenómeno de ELLO, véase Camacho (2013b: \$3). 
heterogéneo dentro de la familia lingüística ibero-románica. ${ }^{6}$ Se verá que el ELLO contemporáneo asume propiedades tanto expletivas como pragmáticas incluso dentro de la misma variedad. A lo largo de este artículo, veremos cómo la distribución estructural heterogénea que se observa entre las variedades en las que ELLo se usa y de hecho instancia las distintas etapas de pragmatización alcanzadas por ELlo en cada variedad. Así, la distribución y función actual de ELLO se entiende mejor en términos de su desarrollo diacrónico. Resaltamos la importancia de examinar el estatus sintáctico de ELLO como supuesto sujeto expletivo tanto desde la perspectiva sincrónica como la diacrónica, ya que en ambos casos hace falta entender cómo puede surgir un sujeto expletivo en una lengua de sujeto nulo, en primer lugar.

\section{DATOS EMPíricos SINCRÓNICOS}

El análisis que se presenta aquí se sustenta en datos recogidos de hablantes nativos de las lenguas ibero-románicas, así como varias fuentes textua-

${ }^{6}$ En el sentido de que lo que se dice de una variedad respecto a la distribución y función de ELLO no se aplica necesariamente a la caracterización de ELLO en otras variedades. Por ejemplo, la presencia de ELLO en oraciones subordinadas en una determinada variedad no supone su atestación ni su gramaticalidad en otra variedad parecida. Notamos abajo, por ejemplo, algunos contrastes entre los datos del portugués europeo dialectal recopilados en Carrilho (2005) y los obtenidos a través de entrevistas con hablantes nativos en el presente trabajo. En los trabajos anteriores, existe una tendencia hacia la aplicación (explícita o implícita) de una caracterización descriptiva y/o teórica de un conjunto de datos a todas las variedades íbero-románicas en que se da el fenómeno de ELLo. En contraste, aquí resaltamos que tal posición es insostenible y que, en su lugar, resulta más aconsejable evaluar caso por caso el estatus de ELLo en cada variedad para así lograr una caracterización más fiel a los hechos empíricos. 
les, la mayoría de las cuales están disponibles en la red. ${ }^{7}$ Adoptamos una perspectiva cartográfica (Rizzi 1997; 2004; Cinque 1999; Cinque \& Rizzi 2010), marco teórico que mapea la relación entre la interpretación semántico-pragmática de una oración y su representación sintáctica, conforme a una jerarquía funcional universal, dentro de la cual se postula una correspondencia uno-a-uno (idealizada) entre interpretación y proyección funcional. Así pues, los hablantes de las variedades ibero-románicas estándar rechazaron el uso de ELLo como se ha reportado en, entre otros, Uriagereka (1995; 2004; 2005), Silva-Villar (1998), Carrilho (2005; 2008), Kaiser (2006), Hinzelin \& Kaiser (2006; 2007), Martínez-Sanz (2007; 2011), Martínez-Sanz \& Toribio (2008), Bullock \& Toribio (2009), Hinzelin (2009), Bartra-Kaufmann (2011), Kaiser, Oliviéri \& Palasis (2013), Camacho (2013a; 2013b) y Muñoz Pérez (2014). No obstante, el fenómeno de ELlo se observa sobre todo en Hispanoamérica, mayormente en el habla no estándar de la República Dominicana y otros países caribeños, así como en algunas variedades no estándar de la Península Ibérica. Además, se ha verificado la existencia de ELLO en todas variedades ibero-románicas mayoritarias (es decir, el castellano europeo y el latinoamericano; el catalán; y el portugués brasileño y el europeo) en los textos modernos e históricos, variación que se ejemplifica parcialmente en (7-11):

${ }^{7}$ Los datos fueron recopilados de las siguientes fuentes auténticas (lista no exhaustiva): foros y periódicos on-line, blogs y otras formas de medios sociales, corpus basados en Internet, documentos en línea y fuera de línea y textos literarios. Cuando no se consigne la fuente, se trata de ejemplos obtenidos directamente de mis informantes. 
82

(7) ESPAÑol PUERTORRIQUEÑo

Si ello hay bomba se la activo (2005, Alexis y Fido, No lo dejes que se apague, canción)

(8) EsPañol dominicano

Ello hay uno por ahí que se cree que la suya va de cabeza pal top 3... (2012, foro Internet)

(9) Gallego

Entón, el sera verdade que o centro fala e a periferia non responde?

'Entonces, $(e l)$ será verdad que el centro habla y la periferia no responde?' (Google, 2008, Iglesias, Novos retos para a propiedade intelectual)

(10) Español MeXicano (siglo Xix)

Ello es cierto que malos maestros pueden dar buenas lecciones. (CdE, 1802, Fernández de Lizardi, Periquillo Sarniento)

(11) Catalán (siglo Xix)

Ell es veritat que permeté fos saciát de oprobis, burlas y escarnis.

'(Ell) es verdad que permitió saciarse de oprobios, burlas y escarnios.' (Google, 1834, Josep Antoni Arnautó, Manual de piadosas meditacions en catalá)

Aun así, tras realizar entrevistas con hablantes de varios países y regiones, solo recibimos confirmación positiva de ELLO en las siguientes variedades ibero-románicas: el catalán balear, el español dominicano (de la 
región del Cibao) y el portugués europeo de Trás-os-Montes. Por ello, en lo sucesivo, se hace uso de los juicios de gramaticalidad obtenidos de las hablas de dichos entrevistados. Hemos de resaltar que los datos que resumimos abajo reflejan solo los obtenidos en la presente investigación, por lo que no siempre encuadran con las observaciones sobre ELLO de otros autores (de ahí la afirmación de que se trata de un fenómeno heterogéneo). ${ }^{8}$ Así pues, según las pruebas empíricas recogidas durante la reunión de datos de esta investigación, verificamos que ELLO aparece en una serie de construcciones típicamente expletivas (\$3.1) a la vez que manifiesta diversas propiedades relacionadas con el discurso (\$3.2). Las evidencias presentadas a continuación indican que el ELlo actual no debe considerarse problemático para nuestro entendimiento del parámetro de sujeto nulo ni tampoco para su tipología.

8 A título de ejemplo, notamos que el ejemplo (28), cuya exposición se adelanta aquí en (iv), contrasta con los datos de Carrilho (2005) respecto a las interrogativas-qu:

(iv) *Ele que raios fazes aqui?!

(‘¡i(ELLO) qué rayos haces aquí?!')

(v) Ele que raio de gente come estes bolos? - Ninguém.

'(ELLO) quién rayos come estos pasteles? - Nadie.' (Carrilho 2005: 172)

Como supondría una tarea enorme la de delimitar la gran cantidad de variación que existe entre los dialectos ibero-románicos no estándar en los que se encuentra el fenómeno de ELLO, nos limitamos aquí a exponer los datos empíricos obtenidos a lo largo de la presente investigación. Para obtener un panorama más complejo de la gran variación observada en la distribución y función de ELLo, sugerimos que el lector se dirija a otros trabajos sobre el fenómeno, sobre todo los de Carrilho (2005; 2008), Silva-Villar (1998), y Hinzelin \& Kaiser (2006; 2007). 


\subsection{Características expletivas}

ELLO se da en las construcciones impersonales existenciales (12), de extraposición (13) y atmosféricas (14) en todas las variedades ibero-románicas aquí investigadas:

(12) Español dominicano

Ello hay un tro de vaina buena y un tro de vaina mala.

(13) Portugués europeo (Trás-os-Montes)

Ele é sabido o Mário gosta de dançar.

'(ELLO) es sabido que a Mario le gusta bailar.'

(14) Catalán balear

Ell farà més fred que mai aquest hivern.

'(ELLO) hará más frío que nunca este invierno.'

Aparece también en construcciones con verbo inacusativo, en las cuales ELLO ocupa una posición pre-verbal (15); de pasivo (16); de se-impersonal (17) y en las oraciones copulativas (18):

(15) Portugués europeo (CÂmara de Lobos, Funchal)

Ele vem o dinheiro aí mas para donde é que vai o dinheiro? Ninguém vê. '(ELLO) viene el dinero de allí pero ¿a dónde va el dinero? Nadie lo ve.' (Cordial-Sin CLC02) 
EL CAMBIO LINGÜÍSTICO Y LA INTERFAZ SINTAXIS-PRAGMÁTICA

(16) EsPañol dominicano

Ayer hubo un motín. Ello fueron detenidos dos estudiantes.

(17) Portugués europeo (Ribeiras, Horta)

Estando fraca, a gente já vai experimentando, ele vão-se habituando àquilo.

'Siendo débil, la gente ya va experimentando, (ELLO) se va acostumbrando a aquello' (Cordial-Sin PIC16)

(18) Portugués europeo (Unhais da Serra, Castelo Branco)

Ele era umas dores de cólica enorme.

'(ELLO) eran unos calambres horribles!' (Cordial-Sin UNS44)

El uso de ELlo se da con verbos modales de ascenso (véase Cinque 2004; 2006), solo en el caso de que su complementante no finito sea impersonal:

(19) Portugués europeo (Trás-os-Montes)

Ele deve ser tarde já.

'(ELLO) debe de ser tarde ya'

(20) Portugués europeo (Trás-os-Montes)

*Ele deve acompanhá-lo para casa.

('(ELLO) debe acompañarlo hasta su casa’)

La presencia (o imposibilidad) de ELLO en estos casos parece venir determinada por el verbo más bajo, lo cual sugiere que la selección y el 
posterior uso de ELLO sí se rige por factores gramaticales, viz. las propiedades temáticas del predicado más bajo.

Las observaciones de esta sección se resumen en la siguiente tabla:

Tabla 1. Características 'expletivas' de ello

\begin{tabular}{lc}
\hline CONSTRUCCión & ATESTACIÓN DE ELLO \\
\hline Existencial & $\checkmark$ \\
De extraposición & $\checkmark$ \\
Atmosférica & $\checkmark$ \\
Con verbos inacusativos & $\checkmark$ \\
Pasiva & $\checkmark$ \\
Se-impersonal & $\checkmark$ \\
Copulativa & $\checkmark$ \\
Con verbos de ascenso & $\checkmark$ \\
\hline
\end{tabular}

\subsection{Caracteristicas pragmáticas}

A pesar de su apariencia expletiva, se verifica que el elemento ELLo posee varias características relacionadas con el discurso. A saber, el uso de elLo nunca es obligatorio (aunque sí ocasiona un cambio de interpretación), exhibe una asimetría entre oración raíz y subordinada, y se ve asociado con un determinado valor pragmático-semántico.

El expletivo ibérico se manifiesta, pues, en contextos relacionados con la aserción, concretamente en las cláusulas principales (según lo ilustrado arriba) y los complementantes subordinados de verba dicendi (cf. Hooper \& Thompson 1973; Aelbrecht et al. 2012): ${ }^{9}$

9 Según los juicios de gramaticalidad de los hablantes nativos. No encontramos ejemplos 
(21) Español dominicano

Dicen que ello hará mucho frío en este diciembre.

(22) Portugués europeo (Trás-os-Montes)

Acho que ele há uma aranha no banho!

¡Creo que (ELLO) hay una araña en el baño!’

En las estructuras condicionales, observamos un contraste entre las llamadas condicionales premisa ("premise conditionals") y las condicionales evento ("event conditionals"):

Portugués europeo (Alentejo), condicional premisa

pois que prêstimo teem agora as talhas, se êle não há já vinhas?

'Pues para qué sirven las tallas si (ELLO) ya no hay viñas?' (Ribeiro 1927:

28, apud Carrilho 2005: 212)

(24) Portugués europeo (Trás-os-Montes), condicional EVEnto

*Vamos ter que esperar, que não sabemos se ele é fácil chegar à noite.

('Habrá que esperar, que no sabemos si (ELLO) es fácil llegar por la noche')

de ELLo en las cláusulas subordinadas en los datos de habla natural del español dominicano. Aunque para nuestros informantes el uso de ELLo en cláusulas subordinadas solo se daba en contextos relacionados con la aserción, Silva-Villar (1998) sostiene que ELLo es imposible en todo contexto subordinado en las lenguas ibero-románicas del oeste, hallazgo que apoya la hipótesis sostenida aquí de que se trata de un fenómeno raíz. Por el contrario, Carrilho (2005: 211-213) ofrece algunos ejemplos recogidos de dialectos del portugués europeo no estándar en que se halla ELLo en contextos subordinados no relacionados con la aserción. Dejamos para una investigación futura la tarea de determinar de manera más precisa las clases verbales que pueden seleccionar un complementante subordinado con ELLO en las lenguas ibero-románicas. 
Según Haegeman (2003 y trabajos posteriores), la diferencia de gramaticalidad entre (23) y (24) está relacionada con la complexidad del sistema-C, y más concretamente con la presencia y la ausencia de la proyección de Fuerza en los respectivos complementos dependientes. Desde esta perspectiva, el nudo SFuerza codifica la fuerza ilocutiva de aserción, de ahí que el uso de ELLO - permitido en oraciones condicionales premi$s a$ (23), cláusulas que tienen una estructura más periférica, pero no en las evento (24), las cuales están más estrechamente integradas con el núcleo sintáctico de la oración- se ve motivado por factores pragmático-discursivos, y no por la necesidad de llenar la posición del sujeto en el Espec de ST. Es decir, la gramaticalidad de ELLO en las condicionales premisa y la imposibilidad de su uso en las condicionales evento sugiere que ELLO se halla en la proyección adicional de la primera, a saber, el nudo SFuerza, en lugar de una posición más baja, como la asociada con la PPE (véase la nota 4).

Asimismo, la gramaticalidad de ELLO en las oraciones interrogativas viene determinada por la fuerza ilocutiva de la construcción en lugar del tipo de oración. ${ }^{10}$ En el español dominicano y el portugués europeo, se admiten las preguntas polares (interrogativas sí/no) solo en el caso de que i) el elemento ELlo surja en posición inicial de la oración (es decir, queda prohibido invertir sujeto y verbo), y, al menos en el portugués europeo, ii) el interlocutor espere confirmación afirmativa respecto a su solicitud por parte del oyente (o sea, no se trate de una pregunta verdadera):

10 Cf. Coniglio \& Zegrean (2012) para la distinción conceptual/estructural entre el tipo de oración y la fuerza ilocutiva. 
(25) Portugués europeo (Trás-os-Montes)

Ele há carne para comer esta noite?

$\pm ` ¿$ (ELLO) hay carne para comer esta noche, verdad?’

(26) *Há ele carne para comer esta noite?

Observamos que (25) presenta la estructura de una oración interrogativa, mientras que su fuerza ilocutiva tiene un valor más similar de el de una aserción. A la inversa, todas las demás oraciones interrogativas son infelices con ELLO, incluidas las interrogativas-qu (27), así como las preguntas retóricas $(28):^{11}$

(27) EsPañol dominicano

*EEllo por qué vino sin su novia?

(28) Portugués Europeo

*Ele que raios fazes aqui?!

(‘ï(ELLO) qué rayos haces aquí?!’)

Como puede observarse en el contraste entre los ejemplos (25) y (26), el ELLO portugués suele ocupar una posición periférica, incluso inicial, de la oración. En el catalán balear, su posición inicial es obligatoria en todos los contextos, correspondiendo a una proyección muy alta en el sistema

11 En el catalán balear, el uso de ELLo en oraciones interrogativas queda prohibido en todos los casos. 
de C extendido (a partir de Rizzi 1997), concretamente SFuerza. Así, se verifica que el ELLo catalán precede los SDs focalizados (29) y los constituyentes-qu en estructuras exclamativas (30):

(29) Catalán balear

Ell FLORS creixen al sostre, que estrany!

'(ELLO) FLORES crecen en el techo, ¡qué raro!'

(30) Catalán balear

Ell què maca que us ha quedat la foto!

'(ELLO) qué bonita que os ha quedado la foto!'

Sin embargo, no puede preceder ni constituyentes-qu en las interrogativas (31) ni el complementante, por ejemplo en los imperativos de subjuntivo (32):

(31) Catalán balear

*Ell quin temps fa avui?

(‘(ELLO) qué tiempo hace hoy?’)

(32) Catalán balear

*Ell que siguis feliç!

( ‘(ELLO) que seas feliz!')

La diferencia en gramaticalidad de ELLo con los constituyentes-qu entre las frases exclamativas y las interrogativas podría indicar que el 
elemento ELLO tenga propiedades pertenecientes al hablante del enunciado pero no al oyente, abstracciones discursivas que, según ciertos estudios recientes, podrían estar codificadas en distintas proyecciones funcionales de la periferia izquierda (cf. Speas \& Tenny 2003; Giorgi 2010; Sigurðsson 2010 para la representación sintáctica de los referidos rasgos interpretativos en la periferia izquierda oracional). De igual forma, la prohibición sobre la apariencia de ELLO en (32) parece ser una restricción estructural en vez de semántico-pragmática, ya que otros tipos de construcción imperativa (p.ej. Ell anam al cinema!, '¡vamos al cine!'; Ell dóna’m el pa!, 'ipásame el pan!') están permitidos en el catalán balear.

En el caso del español dominicano, ello se da siempre como constituyente inicial de la oración (33), aunque, al someter el elemento a varios diagnósticos distribucionales, no puede anteceder a ningún constituyente periférico, como se ilustra en (34):

(33) Español dominicano

Ello nació un niño el otro día en el pueblo de la tía Maite, ¿̨no lo sabías?

(34) Español dominicano

${ }_{i}$ Ello qué bonita te ha quedado la foto!

En el portugués europeo, ELlo puede darse en dos proyecciones, alternando entre una alta en el sistema C (SEval, debajo de SFuerza pero encima de SEpis) y otra en el frontera ST/SC (SFinitud). ${ }^{12}$ En su posi-

12 Corr (2015: 211-212) aporta pruebas a favor del análisis de ELLo en SFinitud. 
ción periférica, ELLO puede seguir (35) pero no preceder al complementante (36):

(35) Portugués europeo (Trás-os-Montes)

Que ele no fondo não são pessoas más.

'Que (ELLO) en el fondo no son malas personas.'

(36) Portugués europeo (Trás-os-Montes)

*Ele que no fondo não são pessoas más.

Puede también anteceder los adverbios epistémicos (y aquí, irrealis): ${ }^{13}$

(37) Portugués europeo (Trás-os-Montes)

Ele talvez haja melhores opçôes...

'(ELLO) quizás haya mejores opciones...'

Así, la primera posición estructural de ELLO en el portugués europeo sería:

(38) [SFuerza que [SEval ELLo [SEvid aparentemente [SEpis tal vez...

Además, como también aparece abajo de constituyentes topicalizados (39) y focalizados (40), hace falta postular una segunda posición estructural para ELLO en la frontera ST/SC:

13 Cf. las cuarto proyecciones funcionales más altas de Cinque (1999):

(vi) $\mathrm{C}_{\text {Mood }}$ Speech Act $\mathrm{C}_{\text {Mood }}$ Evaluative $\mathrm{C}_{\text {Mood }}$ Evidential $\mathrm{C}_{\text {Mod }}$ Epistemic... 
(39) Portugués europeo (Trás-os-Montes)

As jarras, essas que repartem nas festas, ele há umas na despensa, verdade? '¿Las jarras, esas que reparten en las fiestas, (ELLO) hay unas en la despensa, verdad?'

(40) Portugués europeo (Trás-os-Montes)

TODA A NOITE ele choveu! ‘TODA LA NOCHE (ELLO) llovió!’

Podría parecer que las diferencias respecto a la distribución de ELLO en la periferia izquierda corresponderían a diferencias en su interpretación, predicción que queda comprobada por los informantes nativos, quienes corroboraron las descripciones del valor semántico-pragmático de ELLO tal como se sugiere en trabajos anteriores (Carrilho 2005; 2008; Hinzelin 2006; Hinzelin \& Kaiser 2006; i.a.). Así que, en el catalán balear, el elemento ELLO posee un valor exclamatorio e intensificador (Veny 1989; Bartra-Kaufmann 2011; Nicolau Dols, c.p.), o sea un significado bastante general de interjección, o parecido. En el portugués europeo, sin embargo, el uso de ELLO asume una interpretación mucho más precisa: el compromiso por parte del interlocutor con la verdad de la proposición, así como una creencia en ella (cf. SEvaluación de Cinque 1999; Ambar 2003; Speas \& Tenny 2003), es decir que posee un valor epistémico, perspectiva compartida por Carrilho (2005). ${ }^{14}$ En el caso del español

14 Carrilho (2005; 2008) y Uriagereka (2005) se refieren al valor evidencial de ELLO, pero preferimos aquí emplear el término epistémico, ya que ello no codifica la fuente de información, sino la evaluación de la verdad del enunciado por parte del interlocutor (Aikhenvald 2004 trata la categoría gramatical de la evidencialidad). 
dominicano, disponemos de ejemplos de ELLo en los que desempeña un papel claramente pragmático, como el (41) en que se utiliza ELlo para reforzar la interpretación contrastiva, así como usos en que la interpretación pragmática resulta menos obvia, como el (42):

\section{(41) Español dominicano}

Ello hay una parada frente a la Secretaría. (2006, conversación en que el interlocutor se corrige sobre la ubicación de la Secretaría)

(42) Español dominicano

Ello hay tres películas de un espía llamado Jason Bourne no sé como se llaman si alguien me da el nombre o sube se lo agradecería (2014, Facebook)

Sin embargo, teniendo presente que el uso de ELLO resulta productivo solo en un subconjunto de construcciones impersonales en el tiempo presente en contextos matriz (principalmente ello hay), y que exhibe una distribución oracional restringida, se puede asumir que ello sí goza de algún valor discursivo incluso en el español dominicano, de modo que la interpretación enfática de ELLO pueda entenderse en todos los casos como el refuerzo del compromiso con la verdad del enunciado por parte del interlocutor mediante la fortalecimiento de la fuerza asertiva de la oración. Es más, Henríquez-Ureña, escribiendo en la primera mitad del siglo $\mathrm{xx}$, hace comentarios parecidos sobre el tema, afirmando que "es probable que ello tenga valor enfático [...] y que agregue el matiz que darían fórmulas adverbiales como realmente, en verdad" (Henríquez-Ureńa 1939: 228): en otras palabras, el uso de ELLO a principios del siglo XX 
da lugar a una interpretación epistémica. En suma, la impresión general que se retira del uso de ELlo en las lenguas ibero-románicas es que ELlo refuerza el valor asertivo de una oración, ${ }^{15}$ y que puede, por lo menos en ciertas variedades, conllevar una interpretación epistémica, señalando el compromiso del interlocutor con la verdad del enunciado.

Las características "pragmáticas" de ello y la distribución del elemento por la periferia izquierda se resumen en la Tabla 2:

Tabla 2. Características "pragmáticas” de ello según variedad

\begin{tabular}{lccc}
\hline \multicolumn{1}{c}{ CARACTERÍstica/ } & $\begin{array}{c}\text { ESPAÑOL } \\
\text { DOMINICANO }\end{array}$ & $\begin{array}{c}\text { PORTUGUÉs } \\
\text { EUROPEO }\end{array}$ & $\begin{array}{c}\text { CATALÁN } \\
\text { BALEAR }\end{array}$ \\
\hline Asimetría raíz/subordinada & $\checkmark$ & $(\checkmark)$ & $\checkmark$ \\
Posición periférica/inicial & $\checkmark$ & $\checkmark$ & $\mathbf{x}$ \\
Interrogativas polares & $\checkmark$ & $\mathbf{x}$ & $\mathbf{x}$ \\
Interrogativas-qu & $\mathbf{x}$ & $\mathbf{x}$ & $\checkmark$ \\
Exclamativas-qu & $\mathbf{x}$ & $\mathbf{x}$ & $\mathbf{x}$ \\
Imperativos de subjuntivo & $\mathbf{x}$ & $\mathbf{x}$ & $\mathbf{x}$ \\
¿Precede al complementante que? & $\mathbf{x}$ & $\checkmark$ & $\checkmark$ \\
¿Precede a un adverbio epistémico? & $\mathbf{x}$ & $\checkmark$ & $\checkmark$ \\
¿Precede a un constituyente focalizado? & $\mathbf{x}$ & $\checkmark$ & $\mathbf{x}$ \\
¿Sigue a un constituyente focalizado? & $\mathbf{x}$ & $\checkmark$ & $\mathbf{x}$ \\
¿Sigue a un constituyente topicalizado? & $\mathbf{x}$ & $\checkmark$ & $\checkmark$ \\
Valor interpretativo & $(\checkmark)$ & $\checkmark$ & $\checkmark$ \\
\hline
\end{tabular}

15 Otra posibilidad sería interpretar la codificación sintáctica de la aserción del hablante de su postura epistémica (viz. su creencia en la verdad de la proposición) como la atenuación de la fuerza asertiva (Munaro 2009: 153), pues es una verdad subjetiva, no objetiva, la que enfatiza el hablante. 


\subsection{Repercusiones paramétricas}

Debido a las varias características pragmáticas manifestadas por ELLo, las cuales sitúan el elemento dentro de la categoría de fenómenos de cláusula principal ("main clause phenomena", cf. Aelbrecht et al. 2012), este llamado expletivo "visible" no puede equipararse a los sujetos expletivos semánticamente vacíos como los observados en las lenguas de sujeto no nulo. ${ }^{16}$ En este sentido, el expletivo ibérico investigado aquí no plantea un serio desafío a la fuerte generalización empírica del parámetro de sujeto nulo respecto a la prohibición de los sujetos expletivos visibles. No obstante, si queremos considerar ELLO como un tipo de expletivo visible - propuesta que podría resultar razonable, dadas sus muchas características expletivas- habrá que reconsiderar la tipología de expletivos, ampliándola para así englobar el rango de los matices de los datos empíricos (Biberauer et al. 2009; Biberauer \& Cognola 2014 tratan esta cuestión).

\section{Origen de ello}

Ya hemos sugerido que la variación observada entre las variedades en las que el expletivo ibérico ELlo está hoy atestada, instancia las distintas etapas de pragmatización alcanzada por el elemento en cada variedad. Si conceptualizamos la gramaticalización, y por tanto la pragmatización, como el reanálisis hacia arriba de la estructura funcional en la línea de

16 Una vez más, le recordamos al lector que la presente discusión se limita a los datos empíricos recogidos durante la investigación aquí tratada. 
Roberts \& Roussou (2003), entre otros, se puede decir que la gramaticalización/pragmatización se ve más avanzada en el catalán balear, donde ello lexicaliza una proyección alta en la periferia izquierda; menos avanzada en el español dominicano, donde permanece en una proyección relativamente baja (en la frontera ST/SC); mientras que el portugués europeo proporciona evidencias del cambio lingüístico en curso ya que ELLO parece ensamblarse en dos posiciones: una baja en la periferia izquierda (concretamente, SFinitiud) y otra más alta (SEvaluación). ${ }^{17}$

Ahora bien, las propiedades pragmáticas del expletivo ibérico ELLO plantean la cuestión de cómo este elemento puramente sintáctico llega a convertirse en un elemento pragmático-epistémico. Los trabajos anteriores que tratan su origen histórico (Henríquez-Ureña 1939; Bartra-Kaufmann 2011; Gupton \& Lowman 2013) suponen que el fenómeno actual de ello deriva del uso expletivo del pronombre impersonal de la tercera persona del singular en una etapa previa de la lengua. No obstante, esta hipótesis no explica cómo podía surgir ELlo en una estructura expletiva en una lengua de sujeto nulo en primer lugar. Aquí, en cambio, proponemos que ELLO tiene un origen inesperado en construcciones epistémicas -concretamente, ello es verdad/cierto que- que se evidencian a partir del siglo xv. Se sugiere que estas construcciones se originan mediante el reanálisis de dos cláusulas independientes y yuxtapuestas (sc. ello es ver$d a d$, así como otra frase seleccionada por el complementante que) como una sola oración compleja y subordinada. Esto a su vez entraña la pér-

17 Estas posiciones periféricas se resumen así:

(vii) $\left[\mathrm{SFuerza}_{\mathrm{ELLO}} \mathrm{BalCat}\left[\mathrm{SEval}_{\mathrm{ELLO}} \mathrm{EPalta} \ldots\left[\mathrm{SFin} \mathrm{ELLO}_{\mathrm{DomSp}}, \mathrm{ELLO}_{\mathrm{EPbaja}} \ldots\right.\right.\right.$ 
dida de referencialidad del pronombre ello y su posterior adquisición de matices subjetivos-epistémicos por asociación con el entorno epistémico. En $\$ 4.1$, ofrecemos algunas pruebas a favor del origen epistémico de ELLO, y en $\$ 4.2$, describimos los cambios implicados en la evolución histórica de Ello que avanzamos aquí.

\subsection{ELLO: procedencia impersonal epistémica}

Primero, hemos de admitir que las evidencias diacrónicas de ello son pocas y bastante esparcidas. Los registros efectuados en los corpus históricos del portugués producen muy pocos ejemplos del mismo. No obstante, hay evidencias suficientes de ELLo en los corpus diacrónicos del español que hemos utilizado aquí, ${ }^{18}$ las cuales nos permiten reconstruir el desarrollo diacrónico de ello, por lo menos en la medida en que puede aplicarse a la historia del español. En los demás apartados de esta sección, se resumen los cambios que conlleva la hipótesis del origen epistémico de ELLO.

El caso atestiguado más antiguo de una construcción con uso no referencial de ello en el conjunto de datos aquí investigados remonta al siglo Xv:

(43) La historia tripartita dize lo mismo: empero cuenta que jhesu cristo mismo hablo al emperador sea donde quiera sin mas escreuir otras

18 Principalmente, el Corpus de Español (CdE) de Mark Davies y el Corpus Diacrónico del Español (CORDE) de la Real Academia Española. 
opiniones; ello es verdad que aparecio el dicho seńal: y que lo tuuo despues por bandera. (CORDE, 1498, Ampiés, Tratado de Roma)

Se puede contrastar el ejemplo arriba citado con el uso referencial de ello en un texto contemporáneo:

(44) Pero, Rey mío, todo ello es bueno para poner debajo tus pies. (CdE, 1548, Mal Lara, Recibimiento)

Se verifica que la realización de ELLO en construcciones epistémicas es anterior a su ocurrencia en otros contextos impersonales: en los corpus, las estructuras ello es cierto/verdad que son atestadas a partir de los siglos XV-XVI, aunque las construcciones de extraposición impersonales analógicas (o sea, sin valor epistémico, por ejemplo ello es necesario/visto/fácil que) no aparecen sino hasta los siglos XVII-XVIII. No nos resulta casual que el ELLO actual tenga un significado muy parecido a los ejemplos más antiguos y más asiduos de construcciones diacrónicas con ELLO.

Además, podría esperarse que las construcciones formadas con ELLO reflejasen la frecuencia (o falta de ella) de sus contrapartes sin el presunto expletivo. Observamos que las estructuras existenciales con hay (pero sin ello) son mucho más frecuentes en los corpus que las estructuras epistémicas es verdad/cierto que. Por consiguiente, se podría predecir que la atestación de estructuras con Ello se repetirían el mismo patrón observado en las oraciones homólogas sin ello. No obstante, sucede lo contrario con las construcciones ELLO: las estructuras ello es verdad/cierto que 
aparecen en los corpus con relativa frecuencia mientras que, por otra parte, se registran muy pocos ejemplos de la construcción existencial ello hay y las construcciones de extraposición con ELLO no epistémicas. Esta atestación desigual e inesperada puede explicarse si el fenómeno de ELLO se origina en el antedicho entorno epistémico, difundiéndose a otros contextos posteriormente.

\subsection{Desarrollo histórico}

La hipótesis que presentamos es que la construcción ello es verdad que es una innovación que se deriva del reanálisis de dos cláusulas independientes: la cláusula matriz y referencial ello es verdad (una innovación en sí, compuesta por el sujeto referencial ello y el predicado es verdad) y otra cláusula independiente seleccionada por el complementante que. La yuxtaposición de las dos frases establece un vínculo pragmático entre ellas, relación paratáctica que se ve reanalizada como una subordinada. Los cambios diacrónicos propuestos aquí se resumen en lo siguiente:

(45) a. Existencia de dos estructuras distintas (ello con valor referencial): [ello $\mathrm{REF}_{\mathrm{REF}}$ es verdad]; [es verdad que...] $\left({ }^{*}\left[\mathrm{ello}_{\mathrm{REF} / \mathrm{NO}-\mathrm{REF}}\right.\right.$ es verdad que....])

b. Existencia de dos estructuras independientes [ello $\mathrm{o}_{\mathrm{REF}}$ es verdad], [que...] en yuxtaposición pragmática: [ello ${ }_{\text {REF }}$ es verdad] [que...] 
El Cambio lingüístico y la interfaz Sintaxis-pragmática IOI

c. Reanálisis de la relación pragmática como relación subordinada: [ello es verdad] [que...]; [[ello es verdad][que...]]

d. Existencia de tres estructuras distintas (ello sin función referencial en la construcción impersonal): $\left[\right.$ ello $_{\mathrm{REF}}$ es verdad]; [es verdad que...]; [ ello $_{\mathrm{NO}-\mathrm{REF}}$ es verdad que...]

En la primera fase, las dos construcciones -la construcción biclausal y impersonal es verdad que (46-47) y otra construcción epistémica y monoclausal en que ello tiene valor referencial (48) - se encuentran en distribución complementaria:

(46) Es verdad que tienen algún indio, mas el negocio consiste en el christiano. (CdE, 1500, Colón)

(47) Y si murió y padeció Cristo para enseñarnos lo que cuesta un pecado y lo que hemos de huirle, ¿de dónde coliges que murió para darnos licencia para hacer delictos? Que satisfizo por todos es verdad: ¿̨luego no tenemos que trabajar nosotros? (CdE, 1612, Quevedo, Los sueños)

(48) Quisieron pues los sabios en la invención destas cosas de los infiernos apartar a los hombres de las maldades: lo cual si así fuese creído, como ello es verdad, en todas las edades hubiera habido pocos malos. (CdE, 1554, Pérez de Moya, Philosofía secreta de la gentilidad)

En esta primera fase, no se evidencian construcciones con ello no referencial. En la segunda fase, sin embargo, empezamos a observar cons- 
trucciones epistémicas ambiguas, en las que el estatus gramatical de ello no queda claro:

(49) Un sabio dijo que la memoria es el pulso del amor, porque tanto más veloz, agudo y continuo anda el pensamiento revolviendo lo que ama, cuanto es más crecido su amor. Y así es ello verdad, que a poco amor hay poca memoria; y a mucho, mucha; y a ninguno, ninguna. (CORDE, 1595, de los Ángeles, Diálogos de la conquista del reino de Dios).

(50) Y créame vuesa merced que esto es lo más acertado; que allí está el cocinero cojo que me adora, y todas las veces que entro a velle, que no son pocas, me hinche un gran plato de carne friática, que en her así, me la espeto como quien se sorbe un huevo; y él no hace sino reír de ver la gracia y liberalidad con que como, que es para dar mil gracias a Dios. Ello es verdad, que anoche uno destos señores pajes o pájaros, o qué son, me dijo que sorbiese una escudilla de caldo que traía en la mano, porque me daría la vida, después de Dios. (CORDE, 1614, Fernández de Avellaneda, Don Quixote)

En los ejemplos de arriba, resulta difícil precisar la referencia del pronombre ello ya que el contexto sintáctico parece ofrecer dos posibles interpretaciones: a saber, el pronombre ello se refiere a la oración anterior (p.ej. un sabio dijo...) o puede que carezca de valor referencial, en otras palabras, sería un sujeto impersonal. En el primer caso, la frase encabezada por el complementante que se encontraría en relación paratáctica con la cláusula asociada (p.ej. y asi es ello verdad, que...), por contrario en el segundo 
quedaría en relación sintáctica y, como tal, dependiente. Es esta situación ambigua la que ofrece las condiciones adecuadas para el reanálisis de la relación pragmática, por lo que suponemos que en algún momento los hablantes reanalizan el vínculo pragmático y asociativo como uno subordinado, aunque en la superficie la oración no sufra alteración visible.

Si queremos defender esta hipótesis, una pregunta inmediata es si el complementante que puede empezar una frase independiente en esta época. ${ }^{19}$ La respuesta parece ser afirmativa, a juzgar por las oraciones a seguir, en las cuales el elemento que se da en contextos autónomos (51) e incluso en oraciones matriz (52):

(51) No hay que cansarte; que no quiero ser tu dama. (CdE, 1628, Ruiz de Alarcón y Mendoza, El desdichado en fingir)

(52) Allí, digo, en el Cielo, se consumará del todo este Sacramento, no solo comunicando su espíritu y vida a las almas, sino su cuerpo, calidades espirituales y dotes gloriosas a los cuerpos de todos. Que esas esperanzas alentaban tanto al Apóstol en otra parte: Unde etiam expectamus Salvatorem Dominum nostrum Jesum Christum, qui reformavit corpus humilitatis nostrae configuratum corpori claritatis suae. (CdE, 1606, Paravicino y Arteaga, Oraciones evangélicas)

Por último, llegamos a un cierto punto alrededor del siglo XviII en que el uso de ello no solo deja de ser ambiguo, sino que siempre asume un

19 Frases independientes como estas se dan con menos frecuencia en el portugués y el catalán. Aun así, tales usos, y otros parecidos, sí se manifiestan (y resultan gramaticales). 
valor no referencial en estas construcciones, por lo cual podemos considerar que el proceso de reanálisis está acabado:

(53) Ello es verdad que no tenemos relox; pero a fe que ha jurado el curo que el primer año santo que venga, tenemos que her unos riquísimos órganos. (CORDE, 1614, Fernández de Avellaneda, Don Quijote)

(54) Ello es cierto que, por falta de gente, y por la decadencia de la agricultura y comercio, estaba Espańa entonces precisada a surtirse del extranjero. (CORDE, 1777, Jovellanos, Carta a Rodríguez Campomanes)

(55) Ello es verdad que las truchas no se pescan... (CORDE, 1841, Bretón de los Herreros, Dios los cría y ellos se juntan)

5. EXTENSIÓN

Proponemos que este uso no referencial de ello extiende a otros contextos a partir del siglo xviı por el proceso de analogía (cf. Harris \& Campbell 1995; Hopper \& Traugott 2003; Narrog \& Heine 2011). Encontramos ejemplos de ello no referencial en construcciones impersonales extrapuestas (56-57) y con verbos de ascenso (58) que, contrario al contexto original, no son epistémicas:

(56) Ello es regular que tengas, ayudada de mi hermana tu amiga y tu consejera, buena porción de mentiras y de embolismos dispuesta para el caso; 
El Cambio lingüístico y la interfaz Sintaxis-pragmática IOS

pero ya conozco todas sus tretas y las tuyas. (CdE, 1786, Fernández de Moratín, El viejo y la niña)

(57) En fin: ello es preciso que pueda haber cuestión, pues si ganamos, todo será pecado venial, y si perdemos, ya apelaremos para tribunal y jueces que nos sepan dar la razón. (CdE, 1833, Estébanez Calderón, Escenas andaluzas)

(58) Ello parece que nuestros amigos los Franceses no son los más delicados del mundo sobre este artículo, cuando esta materia se toma por asunto de risa. (CdE, 1742, Feijoo, Cartas eruditas y curiosas)

Lo encontramos además en construcciones impersonales pero esta vez existenciales, concretamente con el verbo haber:

(59) Ello hay virgen, hay clausura, hay un dios que visita a la doncella, sea por lo que fuere; que eso no nos toca a nosotros averiguarlo. (CORDE, 1758, Isla, Fray Gerundio de Campazas)

Es plausible que ELLO se extienda por analogía a construcciones existenciales con haber debido a su estatus impersonal, pero ¿por qué no se dan ejemplos de ELLO en otras estructuras analógicas, como las construcciones impersonales meteorológicas o las oraciones inacusativas, en el corpus histórico? Una explicación posible es un paralelismo que existe entre, por una parte, las estructuras extrapuestas y las existenciales con haber y, por la otra, las siguientes construcciones monoclausales 
y referenciales: ellolestoleso es verdad/importante; ello/estoleso parece; ellol estoleso hay. Tal analogía no puede aplicarse a las estructuras atmosféricas (*ellolesto/eso llueve) ni las inacusativas (*ellolestoleso viene un chico). Por eso, sostenemos que ELLo no puede extenderse a otros contextos impersonales como los considerados en el $\$ 3.1$ hasta que se estableciera en los entornos sintácticos más parecidos a la construcción original, es decir las estructuras de extraposición y las existenciales.

¿De dónde proviene la interpretación epistémica? En este análisis, asumimos que ELLO obtiene matices semánticos-pragmáticos por el proceso de la llamada "inferencia pragmática", la cual genera significado a través del uso de una forma lingüística en un determinado contexto (Nicolle 2011). Así pues, el uso de la construcción impersonal es verdad que implica el compromiso con la verdad por parte del interlocutor. Es decir, a pesar de ser impersonal y no epistémica, el empleo de dicha frase produce una interpretación necesariamente epistémica, ya que al afirmar que algo es verdad se expresa que uno cree que este "algo" es verdad. Es esta yuxtaposición de ello con el contexto epistémico la que facilita el proceso de transferencia semántica de la interpretación epistémica; en otras palabras, debido a la influencia asociativa de su entorno semántico-pragmático, se da un proceso de subjetificación del elemento ello (cf. Traugott 1995; Langacker 2006), pues la actitud subjetiva del hablante se ve gramaticalizada en el pronombre ello.

Desde la perspectiva generativa, este cambio se asocia con la presencia de rasgos de primera persona codificados en los extremos de la periferia izquierda de la arquitectura oracional (Speas \& Tenny 2003; Giorgi 2010; Sigurðsson 2010). Por eso, preferimos hablar de la "pragmatización" de 
ello en vez de su "gramaticalización", ya que el elemento se desarrolla una interpretación pragmática (viz. el valor epistémico) durante el transcurso de las permutaciones arriba propuestas. Somos agnósticos respecto a los mecanismos sintácticos específicos que estén implicados en dicho proceso, pero notamos que puede suponer la adquisición de rasgos sintácticos (frente a su pérdida durante el proceso de gramaticalización, cf. Roberts \& Roussou 2003), concretamente los relacionados con la deixis y los actos de habla. Sea cual sea la postura correcta respecto a lo anterior, para volver al tema que nos ocupa, hace falta preguntar si en verdad se puede observar esta presunta interpretación epistémica de ello en los datos históricos. Sobre este punto, notemos el uso de ELLO con valor pragmático-epistémico en el siguiente texto histórico:

(60) Pero, ¡aquí está el busilis!: ¡Quántos gramáticos se encuentran que no llegan ni con cien leguas a la dignidad de estos y que les sobrepujan en muchos millares en lo que toca a arrogancia y amor propio! Ello es cierto y muy cierto que todos debieran ser doctos lo más que pudiesen [...] (CdE, Forner, 1776, Los gramáticos: historia chinesca)

El análisis de ELLO como elemento epistémico-pragmático nos permite explicar algunos usos actuales de ELLO que de lo contrario hubiera sido sorprendentes o incluso inexplicables, como se ilustra en los siguientes ejemplos:

(61) Español dominicano

A: ¿Vienes a la fiesta esta noche?

B: Ello. [=sí]. 
(62) Portugués europeo (Outeiro, Braganza)

Mas esse já conhecem, ele já?

'Pero eso ya lo saben, no?' (Cordial-Sin OUT40, apud Carrilho 2005: 134)

Estos ejemplos van más allá del sentido epistémico de ELLO, el cual va asumiendo matices interpretativos cada vez más amplios. En (61), la respuesta ello quiere decir 'sí', o sea que constituye una expresión afirmativa de la creencia del interlocutor. De manera similar, el hablante en (62), mediante el uso de ele já, crea la expectativa de que el oyente ratifique luego la proposición ya establecida (la cual queda establecida en primer lugar por la realización de la pregunta por parte del hablante). Se nota además que las preguntas etiqueta suelen comprender alguna expresión o algún matiz relacionado con el concepto de la verdad (cf. español ¿verdad?, alemán nicht wahr?, inglés right?, italiano (nev)vero?). La comprobación de ELLO en los contextos arriba brevemente descritos constituiría una extensión lógica de la función de ELLo si de verdad este tiene -o en una etapa ha tenido-valor epistémico.

\section{Comentarios finales}

A primera vista, el expletivo ibérico puede parecer problemático para nuestra concepción del parámetro de sujeto nulo, pues al aparecer en contextos expletivos prototípicos, motiva un análisis como elemento análogo al sujeto expletivo "verdadero" de las lenguas de sujeto no nulo como el inglés o el francés. No obstante, al examinar sus características sintácticas e interpretativas, resulta que el expletivo ibérico 
es de una naturaleza bien diferente a la de los expletivos puramente sintácticos, los cuales sirven de mecanismo de último recurso para llenar la posición canónica del sujeto. En cuanto a si debemos por tanto ampliar la tipología de expletivos para así incluir tales elementos situados en la interfaz sintáctico-pragmática, es una cuestión que dejamos zanjar a los demás.

Si bien quedamos de acuerdo con los trabajos anteriores que proponen un análisis periférico para ELlo (o sea, que el elemento pertenece más al dominio-C que al dominio-T), ${ }^{20}$ pace la mayoría de estos estudios, sostenemos que ELLO no puede ocupar múltiples proyecciones funcionales sincrónicamente, sino que surge en (una o, en el caso del portugués europeo, dos) posiciones estructurales distintas según la variedad en cuestión. En concreto, se ha propuesto que ELLO ocupa una proyección alta en la periferia izquierda (SFuerza) en el catalán balear; una posición baja (SFinitud) en el español dominicano; y, por último, una posición alta (SEvaluación) y otra baja (SFinitud) en el portugués europeo. Aunque solo hemos podido esbozar a grandes rasgos un proceso histórico complicado, hemos pretendido demostrar que la variación actual es consecuencia de las distintas etapas de pragmatización alcanzadas por ello en las respectivas lenguas ibero-románicas que admiten su empleo. Las evidencias diacrónicas indican que el presunto expletivo ibérico se origina en contextos impersonales y epistémicos, hipótesis que explicaría el desarrollo del elemento desde sus inicios como sujeto pronominal

20 Cabe señalar que no siempre es nítida la frontera entre los dos dominios (y por lo tanto aún queda por determinar de manera definitiva). 
referencial, pasando por su transformación en un marcador de valor epistémico-pragmático, y finalmente hasta su estado actual en que ELLO mantiene una caracterización diversa, los pormenores de la cual parecen siempre remontarse a la explicación que delineamos arriba.

\section{REFERENCIAS}

\section{Fuentes}

Davies, Mark. 2002-. Corpus del Español: 100 million words, 1200s1900s. http://www.corpusdelespanol.org.

Martins, Ana Maria. (coord.). [2000- ] 2010. CORDIAL-SIN: Corpus Dialectal para o Estudo da Sintaxe / Syntax-oriented Corpus of Portuguese Dialects. Lisboa, Centro de Linguística da Universidade de Lisboa. http://www.clul.ul.pt/en/resources/411-cordial-corpus.

Real Academia Española: Banco de datos (CORDE). [en línea]. Corpus diacrónico del español. http://www.rae.es.

\section{Estudios}

Aelbrecht, Lobke \& Haegeman, Liliane \& Nye, Rachel. (eds.). 2012. Main clause phenomena, vol. 190. Ámsterdam: John Benjamins Publishing.

Aikhenvald, Alexandra Y. 2004. Evidentiality. Oxford: Oxford University Press. 
Ambar, Manuela. 2003. Wh-asymmetries. En Di Sciullo, Anna Maria (ed.), Asymmetry in grammar, vol. I, 209-249. Ámsterdam: John Benjamins Publishing

Andersen, Henning. 1973. Abductive and deductive change. Language 49. 765-793.

Bartra-Kaufmann, Ana. 2011. Recycled neuter expletive pronouns and the nature of the left periphery: ell and relatives revisited. Catalan Journal of Linguistics 10. 185-219.

Biberauer, Theresa. 2009. Semi null-subject languages, expletives and expletive pro reconsidered. En Biberauer, Theresa \& Holmberg, Anders \& Roberts, Ian \& Sheehan, Michelle (eds.), Parametric variation: Null subjects in minimalist theory, 153-199. Cambridge: Cambridge University Press.

Biberauer, Theresa \& Cognola, Federica. 2014. The expletive-impersonal connection: Mòcheno insights into the typology of null-subject languages. (Ponencia presentada en el congreso de Forum for Germanic Language Studies 1, Society for Germanic Linguistics, Universidad de Cambridge, 9-11 enero de 2014)

Biberauer, Theresa \& Holmberg, Anders \& Roberts, Ian \& Sheehan, Michelle. 2009. Parametric variation: Null subjects in minimalist theory. Cambridge: Cambridge University Press.

Bullock, Barbara E, \& Toribio, Almeida Jacqueline. 2009. Reconsidering Dominican Spanish: Data from the rural Cibao. Revista Internacional de Lingüistica Iberoamericana 14. 49-73.

Camacho, José. 2013a. Null subjects. Cambridge: Cambridge University Press. 
Camacho, José. 2013b. On left-peripheral expletives in Central Colombian Spanish. Rutgers University. (Manuscrito inédito.)

Carrilho, Ernestina. 2005. Expletive ele in European Portuguese dialects. Lisboa: Universidade de Lisboa. (Tesis doctoral inédita.) Carrilho, Ernestina. 2008. Beyond doubling: overt expletives in European Portuguese dialects. En Barbiers, Sjef \& Koeneman, Olaf \& Lekakou Marika \& van der Ham, Margreet (eds.), Microvariation in Syntactic Doubling, vol. 36, 301-349. Bingley: Emerald.

Chomsky, Noam. 1957. Syntactic structures. The Hague: Mouton.

Chomsky, Noam. 1981. Lectures on government and binding. Dordrecht: Foris.

Chomsky, Noam. 1982. Some concepts and consequences of the theory of government and binding, vol. 6. Cambridge, Massachusetts: MIT press. Chomsky, Noam. 1986. Knowledge of language: Its nature, origin, and use. New York: Praeger.

Cinque, Guglielmo. 1999. Adverbs and functional heads: A cross-linguistic perspective: Oxford Studies in comparative syntax. Oxford: Oxford University Press.

Cinque, Guglielmo. 2004. Restructuring and functional structure. En Beletti, Adriana (ed.), Structures and beyond. The cartography of syntactic structures, vol. 3, 132-191. Oxford: Oxford University Press.

Cinque, Guglielmo. 2006. Restructuring and functional heads. Oxford: Oxford University Press.

Cinque, Gugilelmo, \& Rizzi, Luigi. 2010. Mapping spatial PPs. The cartography of syntactic structures, vol. 6. Oxford: Oxford University Press. 
Coniglio, Marco \& Zegrean, Iulia. 2012. Splitting up force. En Aelbrecht, Lobke \& Haegeman, Liliane \& Nye, Rachel (eds.), Main clause phenomena: New horizons, vol. 190, 229-256. Ámsterdam: John Benjamins Publishing.

Corr, Alice. 2015. Overt expletives in Ibero-Romance: A diachronic and diatopic perspective. Revue Romaine de Linguistique 60 (2-3). 205-222.

Deutscher, Guy. 2002. On the misuse of the notion of "abduction" in linguistics. Journal of Linguistics 38. 469-485.

Fernández-Soriano, Olga \& Táboas, Susana. 1999. Construcciones impersonales no reflejas. En Demonte, Violeta \& Bosque, Ignacio (eds.), Gramática descriptiva de la lengua española, 1723-1778. Madrid: Espasa Calpe.

Giorgi, Alessandra. 2010. About the speaker: Towards a syntax of indexicality. Oxford: Oxford University Press.

Gupton, Timothy \& Lowman, Sarah. 2013. An F Projection in Cibeño Dominican Spanish. En Cabrelli Amaro, Jennifer \& Lord, Gillian \& de Prada Pérez, Ana \& Aaron, Jessi Elana (eds.), Selected Proceedings of the 16th Hispanic Linguistics Symposium, 338-348. Somerville, Massachusetts: Cascadilla Press.

Haegeman, Liliane. 2003. Conditional clauses: External and internal syntax. Mind \& Language 18(4). 317-339.

Harris, Alice \& Campbell, Lyle. 1995. Historical syntax in cross-linguistic perspective, vol. 74. Cambridge: Cambridge University Press. Henríquez Ureña, Pedro. 1939. Ello. Revista de filología hispánica 1(3). 209-229. 
Hinzelin, Marc-Olivier. 2006. Die neutralen Pronomina «ell», «això», «allò», «açò» und "ço» in unpersönlichen Konstruktionen in diachroner und dialektaler Variation. En Pusch, Claus D., \& Fischer, Susann, La gramàtica pronominal del català: variació-evolució-funció, 46-84. Aachen: Shaker Verlag.

Hinzelin, Marc-Olivier. 2009. Neuter pronouns in Ibero-Romance: Discourse reference, expletives and beyond. En Kaiser, Georg \& Remberger, Eva-Maria (eds.), Proceedings of the Workshop 'Null-subjects, expletives, and locatives in Romance' (Arbeitspapier 123), 1-25. Konstanz: Fachbereich Sprachwissenschaft der Universität Konstanz.

Hinzelin, Marc-Olivier \& Kaiser, Georg. 2006. Das neutrale Pronomen ello im dominikanischen Spanisch und die Nullsubjekteigenshaft. Anhang: Korpus und Bibliographie zu ello und unpersönlichen Konstruktionen (mit Subjekt) im Spanischen. (Arbeitspapier 116). Konstanz: Fachbereich Sprachwissenschaft der Universität Konstanz. http://kops.uni-konstanz.de/handle/123456789/3761

Hinzelin, Marc-Olivier \& Kaiser, Georg, 2007. El pronombre ello en el léxico del español dominicano. En Mihatsch, Wiltrud \& Sokol, Monika (eds.), Language contact and language change in the Caribbean and beyond/Lenguas en contacto y cambio lingüistico en el Caribe y más allá, 171-188. Frankfurt am Main: Peter Lang.

Holmberg, Anders \& Nayudu, Aarti \& Sheehan, Michelle. 2009. Three partial null-subject languages: a comparison of Brazilian Portuguese, Finnish and Marathi. Studia Linguistica 63 (1). 59-97.

Holmberg, Anders \& Nikanne, Urpo. 2002. Expletives, subjects and topics in Finnish. En Svenonius, Peter (ed.), Subjects, expletives, and the EPP, 71-106. Oxford: Oxford University Press. 
Hooper, Joan B. \& Thompson, Sandra A. 1973. On the applicability of root transformations. Linguistic Inquiry 4(4). 465-497.

Hopper, Paul \& Traugott, Elizabeth. 2003. Grammaticalization. Cambridge: Cambridge University Press.

Kaiser, Georg A. 2006. Pronombres sujeto en construcciones impersonales de lenguas iberorrománicas. En Fernández Fernández, Beatriz \& Laka Mugarza, Itziar (eds.), Andolin gogoan. Essays in honour of Professor Eguzkitza, 513-530. Bilbao: Universidad del País Vasco.

Kaiser, Georg. A. \& Oliviéri, Michèle \& Palasis, Katerina. 2013. Impersonal constructions in northern Occitan. En Álvarez Pérez, Xosé Afonso \& Carrilho, Ernestina \& Magro, Catarina (eds.), Current approaches to limits and areas in dialectology, 345-366. Cambridge: Cambridge Scholars Press.

Langacker, Ronald. 2006. Subjectification, grammaticization, and conceptual archetypes. En Athanasiadou, Angeliki \& Canakis, Costas \& Cornillie, Bert (eds.), Subjectification: various paths to subjectivity, 17-40. Berlin: Mouton de Gruyter.

Lightfoot, David. 1979. Principles of diachronic syntax. Cambridge: Cambridge University Press.

Lightfoot, David. 1997. Catastrophic change and learning theory. Lingua 100(1). 171-192.

Martínez-Sanz, Cristina. 2007. Dialectal microvariation and the null subject parameter: The case of Dominican Spanish. University of Ottawa. (Manuscrito inédito.)

Martínez-Sanz, Cristina. 2011. Null and overt subjects in a variable system: The case of Dominican Spanish. University of Ottawa. (Tesis doctoral.) 
Martínez-Sanz, Cristina \& Toribio, Almeida Jacqueline, 2008. On the nature of expletive ello in Dominican Spanish. (Ponencia presentada en el congreso del Hispanic Linguistics Symposium, Université Laval, Quebec.)

Munaro, Nicola. 2009. Toward a hierarchy of clause types. En Benincà, Paola \& Munaro, Nicola (eds.), Mapping the left periphery. The cartography of syntactic structures, vol. 5, 125-162. New York: Oxford University Press.

Muñoz Pérez, Carlos. 2014. Dominican 'ello' as a non-deleted null expletive. Borealis-An International Journal of Hispanic Linguistics 3(1). 155-161.

Narrog, Heiko \& Heine, Bernd (eds.). 2011. The Oxford handbook of grammaticalization. Oxford: Oxford University Press.

Nicolle, Steve. 2011. Pragmatic aspects of grammaticalization. En Narrog, Heike \& Heine, Bernd (eds.), The Oxford handbook of grammaticalization, 142-152. Oxford: Oxford University Press.

Rizzi, Luigi. 1982. Issues in Italian syntax, vol. 11. Dordrecht: Foris.

Rizzi, Luigi. 1986. Null objects in Italian and the theory of pro. Linguistic inquiry 17(3). 501-557.

Rizzi, Luigi. 1997. The fine structure of the left periphery. En Haegeman, Liliane (ed.), Elements of grammar, 281-337. Dordrecht: Springer Netherlands.

Rizzi, Luigi. 2004. Locality and left periphery. En Belletti, Adriana (ed.), Structures and beyond. The cartography of syntactic structures, vol. 3, 223-251. Oxford: Oxford University Press. 
Roberts, Ian. 1993. Verbs and diachronic syntax: a comparative history of English and French. Dordrecht: Kluwer.

Roberts, Ian. 2007. Diachronic syntax. Oxford: Oxford University Press. Roberts, Ian \& Roussou, Anna. 2003. Syntactic change: A minimalist approach to grammaticalization, vol. 100. Cambridge: Cambridge University Press.

Sigurðsson, Halldór Ármann. 2010. On EPP effects. Studia Linguistica 64(2). 159-189.

Silva-Villar, Luis. 1998. Subject positions and the roles of CP. En Schwegler, Armin \& Tranel, Bernard \& Uribe-Etxebarria, Myriam, Romance Linguistics: Theoretical Perspectives. Selected papers from the 27th Linguistic Symposium on Romance Languages (LSRL XXVII), Irvine, 2022 February, 1997, 247-270. Ámsterdam: John Benjamins Publishing. Speas, Peggy \& Tenny, Carol. 2003. Configurational properties of point of view roles. En Di Sciullo, Anna Maria (ed.), Asymmetry in grammar, vol. 1, 315-345. Ámsterdam: John Benjamins Publishing.

Traugott, Elizabeth C., 1995. The role of the development of discourse markers in a theory of grammaticalization. (Ponencia presentada en la XII International Conference of Historical Linguistics, ICHL, Manchester, 13-18 de agosto de 1995.)

Uriagereka, Juan. 1995. An F position in Western Romance. En Kiss, Katalin (ed.), Discourse configurational languages, 153-175. Oxford: Oxford University Press.

Uriagereka, Juan. 2004. A Peripheral Pleonastic in Western Iberian. (Ponencia presentada en el congreso de Expletive Subjects in Romance and Germanic Languages. Konstanz, Germany, noviembre 2004.) 
Uriagereka, Juan. 2005. Iberian pleonasm. University of Maryland. (Manuscrito inédito.)

Veny, Joan. 1989. Els parlars catalans. Sintesi de dialectologia. 8a ed. Mallorca: Editorial Moll.

Walkden, George. 2011. Abduction or Inertia? The logic of syntactic change. En Cummins, Chris \& Elder, Chi-Hé \& Godard, Thomas \& Macleod, Morgan \& Schmidt, Elaine \& Walkden, George (eds.), Proceedings of the Sixth Cambridge Postgraduate Conference in Language Research, 230-239. Cambridge: Institute of Language Research. 